\section{Prematuridade e fatores associados em Santa Catarina, Brasil: análise após alteração do campo idade gestacional na Declaração de Nascidos Vivos}

\section{Premature birth and associated factors in the Brazilian State of Santa Catarina: an analysis after alteration of the gestational age section of the Live Birth Declarations}

Paulo Fontoura Freitas 1

Rafael Rodrigues de Araújo 2 1 Núcleo de Orientação em Epidemiologia. Universidade do Sul de
Santa Catarina (UNISUL). Av. José Acácio Moreira, 787. Humaitá.
Tubarão, SC, Brasil. E-mail: pfreitas.epidemio@gmail.com
2 Departamento de Biologia, Saúde e Ciências Sociais. Universidade
do Sul de Santa Catarina. Tubarão, SC, Brasil.

\begin{abstract}
Objectives: to estimate the prevalence of premature birth and associated factors, after alteration of the gestational age section of Live Birth Declarations in the Brazilian State of Santa Catarina, in 2012.

Methods: a cross-sectional study was carried out based on data from the Live Births Information System (SINASC). Prevalence rates for premature birth, raw and adjusted by Cox's robust regression were calculated for the year 2012 and the results compared with those reported for 2005.

Results: the prevalence of premature birth rose from $6.1 \%$, in 2005, to $10.6 \%$ in 2012. Comparison of the two years found greater differences among nonCaucasian women and those with fewer prenatal consultations and a lower level of schooling. After adjustment for confounding factors, the prevalence of premature birth was found to be associated with a lower frequency of prenatal consultations $(P R=2.64$ CI95\%: 2.58-3.28), lower levels of schooling ( $P R=$ 1.65; CI95\%: 1.11-2.45), maternal age $<20$ years $(P R=1.24$; $C 195 \%: 1.17-1.31)$ and $>39$ years $(P R=$ 1.32; CI95\%: 1.17-1.49) and non-Caucasian (PR=1.14; C195\%: 1.07-1.21.)

Conclusions: changes in the gestational age section, now recorded as full weeks of gestation, have resulted in more reliable measurement of the prevalence of premature birth in Brazil.

Key words Preterm birth, Risk factors, Information systems
\end{abstract}

\section{Resumo}

Objetivos: estimar a prevalência de prematuridade e os fatores associados, após a alteração do campo idade gestacional na Declaração de Nascidos Vivos, no Estado de Santa Catarina, Brasil, em 2012.

Métodos: estudo seccional com base nos dados do Sistema de Informações de Nascidos Vivos (SINASC). Razões de Prevalência (RP) de prematuridade, brutas e ajustadas, por Regressão de Cox Robusta para o ano de 2012, foram calculadas e os resultados comparados aqueles reportados para 2005.

Resultados: as taxas de prematuridade aumentaram de 6,1\%, em 2005, para 10,6\% em 2012. Ao se comparar os dois anos, maiores diferenças foram encontradas entre as mulheres com menor número de consultas pré-natal, menor nivel de escolaridade e de cor não branca. Após o ajuste pelos fatores de confusão, as taxas de prematuridade se mostraram associadas à menor frequência de consultas de prénatal ( $R P=2,64$; IC95\%: 2,58-3,28), menor escolaridade $(R P=1,65 ; I C 95 \%: 1,11-2,45)$, idade materna $<20$ anos $(R P=1,24$; IC95\%: 1,17-1,31) e maior que 39 anos $(R P=1,32$; IC95\%: 1,17-1,49) e de cor não branca $(R P=1,14$; IC95\%: 1,07-1,21.)

Conclusões: mudanças no campo idade gestacional, agora preenchido como semanas completas de gestação, resultaram em medidas mais confiáveis das taxas de prematuridade no Brasil.

Palavras-chave Nascimento prematuro, Fatores de risco, Sistemas de informação 


\section{Introdução}

O aumento nas taxas de nascimento prematuro no âmbito mundial faz com que este desfecho seja considerado mundialmente um dos mais importantes problemas de saúde pública nos dias de hoje. ${ }^{1}$ No Brasil, ainda que uma queda importante nas taxas de mortalidade infantil tenha ocorrido nas últimas décadas, os óbitos neonatais apresentaram um decréscimo bem menos expressivo. ${ }^{2}$ A taxa de prematuridade em 2011, segundo a recente pesquisa "Nascer no Brasil", foi de 11,3\%, tendo este agravo contribuído com mais de $30 \%$ das mortes no período neonatal. ${ }^{3}$

$\mathrm{Na}$ busca do entendimento das razões do aumento temporal nas taxas de prematuridade, diferentes fatores biológicos, sociais e relacionados aos serviços de saúde, incluindo baixo nível socioeconômico e educacional, aumento na proporção de nascimentos entre as mulheres com idade mais avançada e uso crescente da cesárea eletiva sem indicação médica tem sido reportados.4-7

Ainda que os dados do Sistema de Informação sobre Nascidos Vivos (SINASC) para a Região Sul sejam relatados como de razoável confiabilidade, quando comparados a outras regiões do país, ${ }^{8}$ problemas relacionados à forma de coleta dos dados, particularmente aqueles referentes a variável idade gestacional, já vem sendo contestados por estudos que apontam que um crescimento importante nas taxas de prematuridade não vinham sendo detectados pelos dados do SINASC. 4

Em 2011 os dados sobre a idade gestacional, anteriormente coletados como faixas de idade gestacional, passaram a ser registrados em semanas gestacionais inteiras, utilizando como padrão de referência a data da última menstruação (DUM) ou, quando disponíveis, dados de exame físico ou ultrassonografia. ${ }^{9}$ Mesmo que este método ainda esteja sujeito a erros por subestimar a prematuridade quando comparada à ultrassonografia, 10 mudanças na forma da captação da variável idade gestacional resultaram em uma alteração na magnitude da prematuridade no país, passando a ter uma medida mais próxima do que é referido pelos estudos de base populacional no Brasil. 4

O único estudo encontrado na literatura, com dados do SINASC para prematuridade, no Estado de Santa Catarina também reflete estas incongruências mostrando evolução temporais de 5,54\% em 2000 a $6,05 \%$ em 2005,11 abaixo de resultados de estudos de base populacional no mesmo período.

Em um estudo12 publicado em 2013, pesquisadores de 12 universidades desenvolveram um fator para correção da frequência de nascimentos prematuros no Brasil, tendo aplicado este fator à prevalência oficial de nascimentos pré-termo, fornecido pelo SINASC. Os autores concluíram que os dados do referido sistema no período 2000-2010, subestimaram em $40 \%$ a real magnitude das taxas de prematuridade no Brasil, ao comparar as variações apresentadas pelo SINASC (6 e 7\%, de 2000 a 2010) com as estimativas corrigidas, que mostraram valores entre 11 e $12 \%$ para os mesmos anos. Por outro lado, para o ano de 2011, a prevalência de prematuridade reportada pelo SINASC aumentou, ficando $15 \%$ inferior àquela corrigida.

O presente estudo foi conduzido com objetivo de investigar a prevalência e fatores associados à prematuridade em Santa Catarina, tendo como base os dados do SINASC para o ano de 2012, apontando mudanças após a alteração do campo sobre a idade gestacional na Declaração de Nascidos Vivos.

\section{Métodos}

Um estudo com delineamento transversal, baseado em dados secundários, coletou informação do SINASC para o Estado de Santa Catarina (SINASC/SC) para o ano de 2012.13 Para fins da presente análise foram excluídos os partos de gestações com duração inferior a 22 semanas, uma vez que estes incluem um potencial número de abortos e também os partos gemelares, por carregarem uma importante incidência de prematuros.

O desfecho foi definido na forma dicotômica (prematuridade sim ou não). Foram considerados prematuros os nascidos vivos, de parto único, com duração da gestação maior de 22 e inferior a 37 semanas. Para todas as variáveis independentes, as categorias escolhidas como de referência foram as de menor risco esperado (idade 20-29 anos, escolaridade maior que 8 anos completos, estado civil casada, etnia/cor da pele branca, pré-natal com 7 ou mais consultas, parto cesáreo, sexo feminino do recém nascido, multiparidade e ausência natimorto na história pregressa).

Os resultados obtidos para o ano de 2012 foram comparados com dados do SINASC para o ano de 2005, utilizando resultados de outro estudo conduzido com objetivos e métodos semelhantes, ${ }^{11}$ para investigar modificações na relação das variáveis independentes com o desfecho, ao comparar os dois anos.

Uma vez que o estudo de 2005 havia utilizado o odds ratio (OR) como medida de associação, aplicamos o método proposto por Zhang e Yu14 no 
cálculo das razões de prevalência (RP) a partir do OR. Como os dados relativos às razões de prematuridade para 2005 foram obtidos a partir dos dados já publicados, ${ }^{11}$ não foi possível incluir as RP ajustadas nas comparações entre os dois anos, razão pela qual foram utilizadas as medidas brutas.

A existência de significância estatística nas diferenças entre grupos foi calculada pelo teste do qui-quadrado. RP de prematuridade, brutas e ajustadas para confundimento e seus respectivos intervalos de confiança (IC) de $95 \%$ foram estimadas para cada uma das variáveis individuais utilizando a Regressão de Cox Robusta, por fornecer estimativas corretas do risco, sendo uma melhor alternativa na análise de estudos transversais com desfechos binários comparada à regressão logística. 15

A significância estatística das associações foi calculada pelo teste da razão de verossimilhança, com nível de significância de 5\%. Foram incluídas no modelo final as variáveis apresentando um valor de $p<0,10$ e mantidas no modelo aquelas com valor de $p<0,05$. A entrada das variáveis no modelo deu-se de maneira hierarquizada, com as variáveis sociodemográficas, precedendo número de consultas prénatal e por último tipo de parto. O programa SPSS versão 18.0 foi utilizado na análise estatística dos dados.

Este estudo foi aprovado pelo Comitê de Ética em Pesquisa da Universidade do Sul de Santa
Catarina (protocolo 13.068.4.01.III).

\section{Resultados}

De acordo com os dados disponibilizados pelo SINASC ocorreram 89.266 partos de nascidos vivos no Estado de Santa Catarina no ano de 2012, sendo que 87.066 foram nascimentos de partos únicos e destes $9.229(10,6 \%)$ se deram entre recém-nascidos com idade gestacional entre 22-36 semanas.

A Figura 1 apresenta a distribuição das taxas de prematuridade para Santa Catarina no período de 2003-2012 conforme informação fornecida pelo SINASC. Estes resultados apresentam pouca variação nas taxas de prematuridade até o ano de 2010 (6,1\%-7,2\%), iniciando a tendência a taxas mais elevadas a partir de 2011-2012 (9,1\%-10,6\%).

A Tabela 1 apresenta a distribuição dos nascidos vivos de acordo com as variáveis da mãe e do recém nascido. Houve leve predominância dos recémnascidos do sexo masculino. A maioria das mães era de etnia branca, se situavam na faixa etária de 21-39 anos, casadas ou com união estável e com nove ou mais anos de escolaridade, a taxa de cesariana foi de $60,3 \%$ e quase $70 \%$ teve frequência ao pré-natal.

A Tabela 2 apresenta as RP de prematuridade, brutas e ajustadas, de acordo com variáveis da mãe e do recém-nascido. Após o ajuste para confundimento, as variáveis: sexo do recém-nascido, estado

\section{Figura 1}

Taxas de prematuridade (\%) no período de 2003 a 2012. SINASC, Santa Catarina, Brasil, 2012.

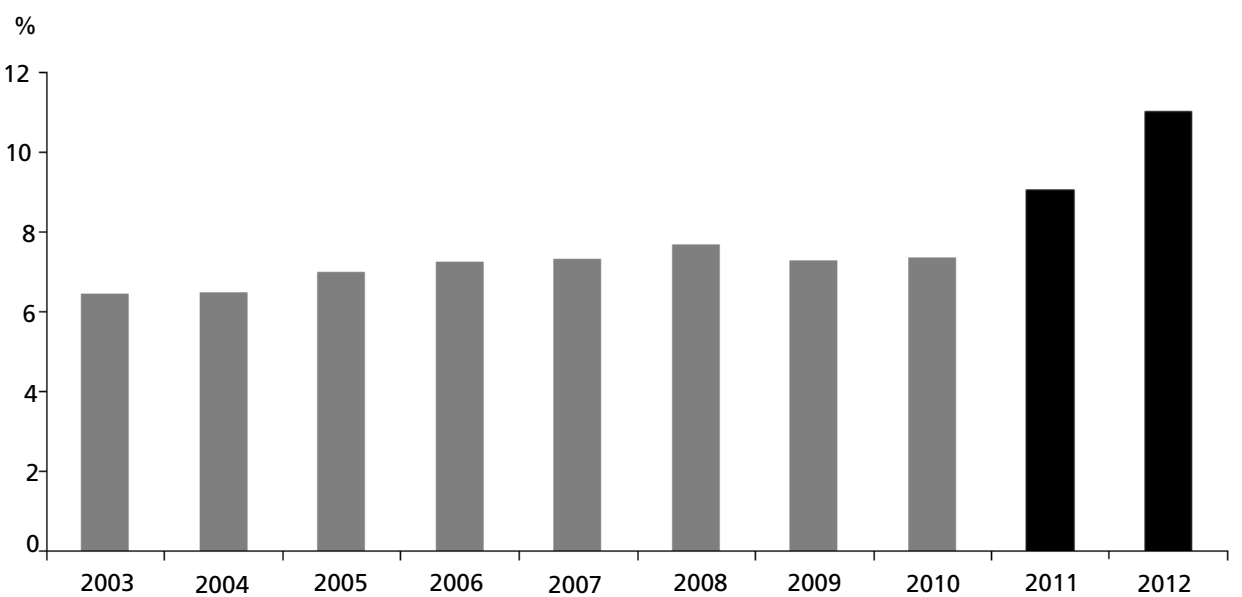

SINASC= Sistema de Informação sobre Nascidos Vivos.

Antes da alteração do campo idade gestacional na Declaração de Nascidos Vivos.

Após alteração do campo idade gestacional na Declaração de Nascidos Vivos. 
civil e tipo de parto, perderam efeito e significância estatística. O resultado da análise multivariada mostrou RP ajustadas, estatisticamente significantes para aquelas mulheres com menor frequência ao prénatal, ou seja, de $0-3$ consultas $(\mathrm{RP}=2,64)$, entre as mulheres nos níveis mais baixos de escolaridade, ou seja, sem educação formal $(\mathrm{RP}=1,65)$, entre as adolescentes $(\mathrm{RP}=1,24)$ e aquelas com idade igual ou superior a 40 anos $(R P=1,32)$, entre as de etnia não branca $(\mathrm{RP}=1,14)$, entre as primíparas $(\mathrm{RP}=$ $1,17)$ e naquelas com história anterior de recém nascido morto $(\mathrm{RP}=1,11)$.

A Tabela 3 apresenta as diferenças nas taxas de prematuridade, ao comparar resultados do SINASC 2005 e 2012. A taxa de prematuridade, entre os nascidos vivos de gestação única, aumentou de
$6,1 \%$, no estudo de 2005 , para $10,6 \%$ em 2012 . A direção do risco ( $\mathrm{RP}>1)$, ao comparar os dois anos, se manteve para todas as variáveis, com exceção de tipo de parto onde a proporção de prematuros em 2012 , ao contrário de 2005 , se mostrou um pouco mais elevada entre as mulheres tendo parto vaginal. As taxas de prematuridade mais elevadas e os maiores acréscimos nestas taxas, ao comparar os dois anos, se deram entre as mulheres no nível mais baixo de escolaridade (de 8,1\% em 2005 para 17,5\% em 2012), entre as mulheres tendo 0-3 consultas no pré-natal (de 15,3\% em 2005 para 20,3\% em 2012) e entre aquelas de etnia/cor da pele não branca, com acréscimo de 6,5\% em 2005 para 12,3\% em 2012.

Tabela 1

Distribuição dos recém nascidos vivos de parto único e dos prematuros de acordo com variáveis da mãe e do recémnascido. SINASC, Santa Catarina, Brasil, 2012.

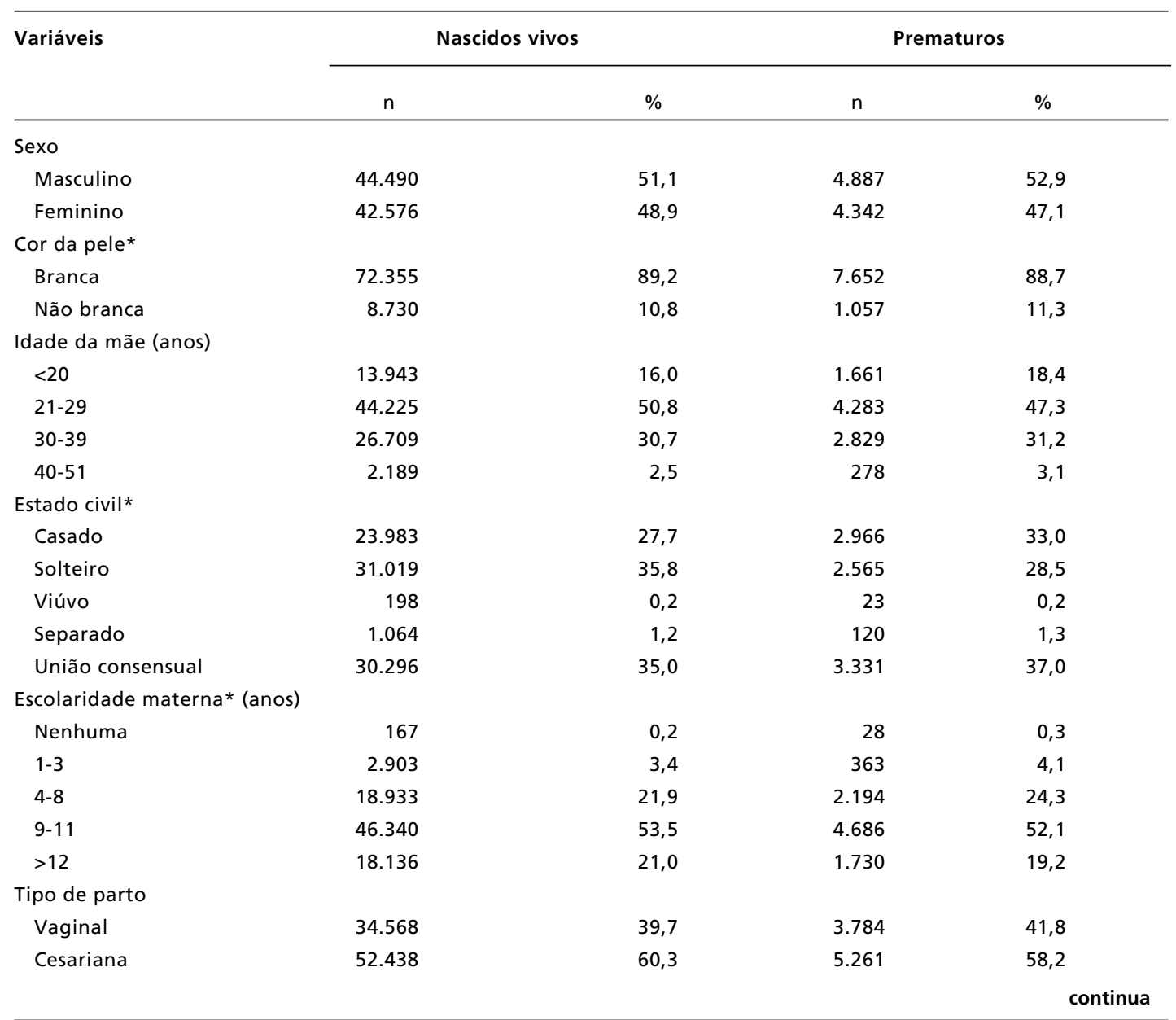

SINASC= Sistema de Informação sobre Nascidos Vivos; * Dados não obtidos para: etnia/cor da pele (6,8\%), estado civil $(0,06 \%)$, escolaridade materna $(0,07 \%)$, consultas pré-natal $(0,03 \%)$ e história de natimorto $(4,5 \%)$. 
Distribuição dos recém nascidos vivos de parto único e dos prematuros de acordo com variáveis da mãe e do recémnascido. SINASC, Santa Catarina, Brasil, 2012.

\begin{tabular}{|c|c|c|c|c|}
\hline \multirow[t]{2}{*}{ Variáveis } & \multicolumn{2}{|c|}{ Nascidos vivos } & \multicolumn{2}{|c|}{ Prematuros } \\
\hline & $\mathrm{n}$ & $\%$ & $\mathrm{n}$ & $\%$ \\
\hline \multicolumn{5}{|c|}{ Consultas no pré-natal* } \\
\hline $0-3$ & 5.321 & 6,2 & 1.083 & 11,9 \\
\hline $4-6$ & 21.133 & 24,3 & 3.449 & 38,0 \\
\hline 7 ou mais & 60.342 & 69,5 & 4.481 & 50,1 \\
\hline \multicolumn{5}{|l|}{ Paridade } \\
\hline Primípara & 37.153 & 42,7 & 4.027 & 44,5 \\
\hline Multípara & 49.913 & 57,3 & 5.024 & 55,5 \\
\hline \multicolumn{5}{|l|}{ Natimorto* } \\
\hline Sim & 13.497 & 16,2 & 1.556 & 17,9 \\
\hline Não & 69.673 & 83,8 & 7.131 & 82,1 \\
\hline
\end{tabular}

SINASC= Sistema de Informação sobre Nascidos Vivos; * Dados não obtidos para: etnia/cor da pele $(6,8 \%)$, estado civil $(0,06 \%)$, escolaridade materna $(0,07 \%)$, consultas pré-natal $(0,03 \%)$ e história de natimorto $(4,5 \%)$.

\section{Tabela 2}

Taxa de prematuridade e razões de prevalência (RP) brutas e ajustadas para prematuridade, de acordo com variáveis da mãe e do recém-nascido. SINASC, Santa Catarina, Brasil, 2012.

\begin{tabular}{|c|c|c|c|c|c|c|}
\hline \multirow[t]{2}{*}{ Variáveis } & \multicolumn{2}{|c|}{ Prematuros } & \multirow[t]{2}{*}{$\mathbf{R P}_{\text {bruta }}$} & \multirow[t]{2}{*}{ IC95\% } & \multirow[t]{2}{*}{$\mathbf{R P}_{\text {ajustada }}$} & \multirow[t]{2}{*}{ IC95\% } \\
\hline & $\mathrm{n}$ & $\%$ & & & & \\
\hline \multicolumn{7}{|l|}{ Sexo } \\
\hline Masculino & 4.887 & 10,9 & 1,07 & $1,04-1,12$ & 1,01 & $0,91-1,08$ \\
\hline Feminino & 4.342 & 10,2 & 1,00 & & 1,00 & \\
\hline \multicolumn{7}{|l|}{ Cor da pele } \\
\hline Branca & 7.652 & 10,7 & 1,00 & & 1,00 & \\
\hline Não branca & 1.057 & 12,3 & 1,15 & $1,08-1,22$ & 1,14 & $1,07-1,21$ \\
\hline \multicolumn{7}{|l|}{ Idade da mãe (anos) } \\
\hline$<20$ & 1.661 & 12,1 & 1,24 & $1,17-1,31$ & 1,24 & $1,17-1,31$ \\
\hline $21-29$ & 4.283 & 9,8 & 1,00 & & 1,00 & \\
\hline $30-39$ & 2.829 & 10,7 & 1,09 & $1,04-1,14$ & 1,08 & $1,03-1,14$ \\
\hline $40-51$ & 278 & 12,9 & 1,31 & $1,16-1,48$ & 1,32 & $1,17-1,49$ \\
\hline \multicolumn{7}{|l|}{ Estado civil } \\
\hline Casado & 2.966 & 9,7 & 1,00 & & & \\
\hline Solteiro & 2.565 & 10,9 & 1,13 & $1,07-1,19$ & 0,94 & $0,89-1,02$ \\
\hline Viúvo & 23 & 11,9 & 1,22 & $0,81-1,84$ & 1,08 & $0,71-1,63$ \\
\hline Separado & 120 & 11,4 & 1,18 & $0,98-1,42$ & 1,04 & $0,87-1,25$ \\
\hline União consensual & 3.331 & 11,2 & 1,15 & $1,1-1,21$ & 1,05 & $0,95-1,06$ \\
\hline \multicolumn{7}{|l|}{ Escolaridade (anos) } \\
\hline Nenhuma & 28 & 17,5 & 1,81 & $1,25-2,63$ & 1,65 & $1,11-2,45$ \\
\hline $1-3$ & 363 & 12,8 & 1,32 & $1,18-1,48$ & 1,26 & $1,13-1,42$ \\
\hline $4-8$ & 2.194 & 11,8 & 1,22 & $1,15-1,30$ & 1,19 & $1,11-1,27$ \\
\hline $9-11$ & 4.686 & 10,2 & 1,06 & $1,05-1,12$ & 1,04 & $0,98-1,11$ \\
\hline$>12$ & 1.730 & 9,7 & 1,00 & & & \\
\hline \multicolumn{7}{|l|}{ Tipo de parto } \\
\hline Vaginal & 3.784 & 11,1 & 1,096 & $1,05-1,14$ & 1,04 & $0,99-1,09$ \\
\hline \multirow[t]{2}{*}{ Cesariana } & 5.261 & 10,2 & 1,00 & & 1,00 & \\
\hline & & & & & & continua \\
\hline
\end{tabular}

SINASC= Sistema de Informação sobre Nascidos Vivos. 
Taxa de prematuridade e razões de prevalência (RP) brutas e ajustadas para prematuridade, de acordo com variáveis da mãe e do recém-nascido. SINASC, Santa Catarina, Brasil, 2012.

\begin{tabular}{|c|c|c|c|c|c|c|}
\hline \multirow[t]{2}{*}{ Variáveis } & \multicolumn{2}{|c|}{ Prematuros } & \multirow[t]{2}{*}{$\mathbf{R P}_{\text {bruta }}$} & \multirow[t]{2}{*}{ IC95\% } & \multirow[t]{2}{*}{$\mathbf{R} \mathbf{P}_{\text {ajustada }}$} & \multirow[t]{2}{*}{$\mathrm{IC} 95 \%$} \\
\hline & $\mathrm{n}$ & $\%$ & & & & \\
\hline \multicolumn{7}{|c|}{ Consultas pré-natal } \\
\hline $0-3$ & 5.321 & 20,4 & 2,72 & $2,61-3,24$ & 2,64 & $2,58-3,28$ \\
\hline $4-6$ & 21.133 & 16,6 & 2,21 & $2,11-2,31$ & 2,25 & $2,15-2,35$ \\
\hline$>6$ & 60.342 & 7,5 & 1,00 & & 1,00 & \\
\hline \multicolumn{7}{|l|}{ Paridade } \\
\hline Primípara & 37.153 & 11,0 & 1,07 & $1,03-1,12$ & 1,17 & $1,11-1,23$ \\
\hline Multípara & 49.913 & 10,2 & 1,00 & & 1,00 & \\
\hline \multicolumn{7}{|l|}{ Natimorto } \\
\hline Sim & 13.497 & 11,7 & 1,13 & $1,07-1,19$ & 1,11 & $1,05-1,18$ \\
\hline Não & 69.673 & 10,4 & 1,00 & & 1,00 & \\
\hline
\end{tabular}

SINASC= Sistema de Informação sobre Nascidos Vivos.

Tabela 3

Diferenças nas taxas de prematuridade, de acordo com as variáveis independentes, ao comparar os dados do para os anos de 2012 e 2005. SINASC, Santa Catarina, Brasil, 2012.

\begin{tabular}{|c|c|c|c|c|c|c|c|}
\hline \multirow[t]{2}{*}{ Variáveis } & \multicolumn{3}{|c|}{$\begin{array}{l}\text { SINASC } 2005 \\
\text { Prematuridade } \\
\quad \mathbf{N}=\mathbf{8 7 . 0 6 6}\end{array}$} & \multicolumn{3}{|c|}{$\begin{array}{c}\text { SINASC } 2012 \\
\text { Prematuridade } \\
\quad \mathbf{N}=\mathbf{8 2 . 5 9 8}\end{array}$} & \multirow[t]{3}{*}{$p^{*}$} \\
\hline & $\%$ & $\mathrm{RP}_{(2005)}$ & (IC) & $\%$ & $\mathrm{RP}_{(2012)}$ & (IC) & \\
\hline Total & 6,1 & & & 10,6 & & & \\
\hline \multicolumn{8}{|l|}{ Sexo } \\
\hline Masculino & 6,2 & 1,05 & $1,0-1,12$ & 10,9 & 1,07 & $1,04-1,12$ & $<0,001$ \\
\hline Feminino & 5,9 & 1,00 & - & 10,2 & 1,00 & - & $<0,001$ \\
\hline \multicolumn{8}{|l|}{ Cor da pele } \\
\hline Branca & 6,1 & 1,00 & - & 10,7 & 1,00 & - & $<0,001$ \\
\hline Nao branca & 6,5 & 1,07 & $0,93-1,25$ & 12,3 & 1,15 & $1,08-1,22$ & $<0,001$ \\
\hline \multicolumn{8}{|c|}{ Idade materna (anos) } \\
\hline 20 & 6,7 & 1,19 & $1,11-1,27$ & 12,1 & 1,24 & $1,17-1,31$ & $<0,001$ \\
\hline $21-29$ & 5,6 & 1,00 & - & 9,8 & 1,00 & - & $<0,001$ \\
\hline $30-39$ & 6,0 & 1,07 & $1,00-1,14$ & 10,7 & 1,09 & $1,04-1,14$ & $<0,001$ \\
\hline $40-51$ & 8,8 & 1,56 & $1,34-1,80$ & 12,9 & 1,31 & $1,16-1,48$ & $<0,001$ \\
\hline \multicolumn{8}{|c|}{ Escolaridade (anos) } \\
\hline Nenhuma & 8,1 & 1,45 & $1,07-1,95$ & 17,5 & 1,81 & $1,25-2,63$ & $<0,001$ \\
\hline $1-3$ & 6,2 & 1,07 & $0,97-1,25$ & 12,8 & 1,32 & $1,18-1,48$ & $<0,001$ \\
\hline $4-8$ & 6,1 & 1,09 & $1,0-1,19$ & 11,8 & 1,22 & $1,15-1,30$ & $<0,001$ \\
\hline $9-11$ & 6,2 & 1,11 & $1,03-1,20$ & 10,2 & 1,06 & $1,00-1,12$ & $<0,001$ \\
\hline$>12$ & 5,6 & 1,00 & - & 9,7 & 1,00 & - & \\
\hline \multicolumn{8}{|l|}{ Tipo de parto } \\
\hline Vaginal & 5,6 & 1,00 & - & 11,1 & 1,11 & $1,05-1,14$ & $<0,001$ \\
\hline Cesariana & 6,6 & 1,18 & $1,12-1,24$ & 10,2 & 1,00 & - & $<0,001$ \\
\hline \multicolumn{8}{|c|}{ Consultas pré-natal } \\
\hline $0-3$ & 15,3 & 3,83 & $3,26-3,85$ & 20,36 & 2,71 & $2,51-3,24$ & $<0,001$ \\
\hline $4-6$ & 8,5 & 2,10 & $1,99-2,22$ & 16,6 & 2,21 & $2,11-2,31$ & $<0,001$ \\
\hline 7 & 4,0 & 1,00 & - & 7,5 & 1,00 & - & $<0,001$ \\
\hline
\end{tabular}

SINASC= Sistema de Informação sobre Nascidos Vivos; * $p$ : qui-quadrado de Pearson para diferenças nas taxas de prematuridade de acordo com ano (2012 e 2005). 


\section{Discussão}

O aumento temporal nas taxas de prematuridade, de registro praticamente mundial, 16 cuja taxa no Brasil atingiu 11,3\% dos nascidos vivos em 2011,3 mantém sua importância como questão prioritária em saúde pública nos dias de hoje. Além de seu papel como principal causa de óbito neonatal e das implicações econômicas para o sistema de saúde, chama atenção que uma considerável proporção dos óbitos entre os bebês prematuros poderia ser evitada por políticas públicas dirigidas a melhoria na assistência ao prénatal, ao parto, e ao recém nascido. ${ }^{17}$ A permanente revisão do SINASC tem alcançado nos últimos anos expressiva melhora na qualidade do preenchimento e apresenta a nível nacional uma cobertura que aproxima os $100 \% .8$ Tem importância central, no presente estudo, a consolidação dos dados do SINASC, levada a cabo em 2011,18 que reporta mudanças na forma de coleta do campo idade gestacional, para semanas gestacionais inteiras, em contraste com faixas de idade gestacional, forma de coleta anterior a 2011.

Ainda que a ultrassonografia (USG) no primeiro trimestre da gestação, padrão ouro para a medida da prematuridade, não esteja representada em grande parte dos dados do SINASC, que utiliza como medida de referência a data da última menstruação, nossos resultados mostram, mesmo assim, taxas de prematuridade com valor próximo aquele referido por estudos de base populacional conduzidos no Brasil. 4 Taxas de prematuridade de 10,6\%, encontradas para o Estado estudado no ano de 2012, aproximam-se às taxas corrigidas para o mesmo Estado no ano de 2011.12

Nossos resultados mostram que as baixas taxas e pequenas variações, de $6,1 \%$ a $7,2 \%$, obtidas a partir dos dados do SINASC para o período de 2003 a 2010, seguidos por uma tendência para taxas mais elevadas a partir de 2011-2012 com 9,1\% e 10,6\%, respectivamente, corroboram a hipótese de uma subestimação nas taxas de prematuridade pelo referido sistema, anterior à consolidação dos dados em 2011.

Da mesma forma, o estudo sugere que alterações na relação das variáveis independentes, classicamente associadas ao desfecho prematuridade, poderiam estar influenciadas, pelo menos em parte, por diferenças na classificação das mulheres quanto à idade gestacional, ao comparar resultados em 2012 com anos anteriores às mudanças na forma de coleta desta variável. Entretanto, é preciso ter em mente que a relação entre melhoria da qualidade da informação e aumento da taxa de prematuridade não é direta, podendo existir outros fatores influenciando o processo. 19,20

Ao comparar nossos resultados com achados de estudo anterior, conduzido com dados do SINASC, para o ano de 2005, 11 buscamos obter informação a respeito de variações a partir do processo de consolidação em 2011.

Diferenças encontradas nas taxas de prematuridade, com um incremento de mais de $40 \%$ de 2005 para 2012, de acordo com dados do SINASC para Santa Catarina, indicam uma magnitude na evolução da ocorrência de prematuridade que não se mantém ao aplicar o fator de correção proposto por Matijasevich et al.,12 que resulta em um aumento bem menor, de 11,1\% em 2005 para 12,2\% em 2012 para o estado como um todo.

Apesar de um aumento temporal nas taxas, a direção do risco $(\mathrm{RP}>1)$ se manteve para quase todos as variáveis, com taxas de prematuridade mais elevadas entre as mulheres de cor da pele não branca, no nível mais baixo de escolaridade, entre aquelas com menor frequência ao pré-natal e nos extremos de idade.

Uma maior prevalência de prematuridade entre as mulheres de nível socioeconômico menos privilegiado, conforme sugerido pelas variáveis escolaridade e cor da pele, vai à direção inversa ao descrito como um paradoxo, por outros autores, aonde prevalências de prematuridade mais elevada são encontradas nas regiões mais desenvolvidas do país (Sul e Sudeste), em comparação com aquelas de menor desenvolvimento (Norte e Nordeste). 12

Em nosso estudo a proporção de prematuros foi um pouco mais elevada entre as mulheres tendo parto vaginal. Ainda que a hipótese de uma associação entre os elevados níveis de prematuridade e o uso crescente da cesariana tenha sido explorada por outros autores, ${ }^{21,22}$ os resultados até aqui são controversos, sendo necessários estudos mais detalhados sobre esta questão.

Nossos resultados, apontando para subestimação nas taxas de prematuridade pelo SINASC, estão de acordo com uma série de outros estudos, $4,10,12$ sugerindo que resultados anteriores ao ano de 2010 sejam interpretados com maior cautela.

Finalmente, apesar da constante evolução na qualidade dos dados do SINASC, revisões do campo idade gestacional, com valorização da ultrassonografia como padrão no preenchimento, têm relevante significado ao levar em conta que a prematuridade é um indicador essencial das condições de saúde perinatal. 


\section{Referências}

1. Beck S, Wojdyla D, Say L, Pilar Betran A, Merialdi M, Requejo JH, Rubens C, Menon R Van Look PFA. The worldwide incidence of preterm birth: a systematic review of maternal mortality and morbidity. Bull World Health Organ. 2010; 88: 31-8.

2. Victora CG, Aquino EML, Leal MC, Monteiro CA, Barros FC, Szwarcwald CL. Maternal and child health in Brazil: progress and challenges. Lancet. 2011; 377: 1863-76.

3. Lansky S, Friche AAL, Silva AAM, Campos D, Bittencourt DAS, Carvalho ML, Frias PG, Cavalcante RS, Cunha AJLA. Pesquisa Nascer no Brasil: Perfil da Mortalidade Neonatal e Avaliação da Assistência à Gestante e ao Recémnascido. Cad Saúde Pública. 2014; 30 (Supl.): S192-S207.

4. Silveira MF, Santos IS, Barros AJD, Matijasevich A, Barros FC, Victora CG. Aumento da prematuridade no Brasil: revisão de estudos de base populacional. Rev Saúde Pública. 2008; 42(5): 957-64

5. Goldenberg RL, Culhane JF, Iams JD, Romero R. Epidemiology and causes of preterm birth. Lancet. 2008; 371: 75-84.

6. Xie R, Gaudet L, Krewski D, Graham ID, Walker MC, Wen SW. Higher cesarean delivery rates are associated with higher infant mortality rates in industrialized countries. Birth. 2015; 42(1): 62-9.

7. Campbell C. Elective cesarean delivery: trends, evidence and implications for women, newborn and nurses. Nurs Women's Health. 2011; 15 (4): 308-14.

8. Frias PG, Szwarcwald CL, Lira PIC. Avaliação dos sistemas de informações sobre nascidos vivos e óbitos no Brasil na década de 2000. Cad Saúde Pública. 2014; 30 (10): 20682280 .

9. Brasil. Ministério da Saúde. Departamento de Análise da Situação de Saúde. Secretaria de Vigilância em Saúde. Manual de instruções para o preenchimento da Declaração de Nascido Vivo. Brasília, DF; 2011.

10. Pereira AP, Dias MA, Bastos MH, Gama SG, Leal MC. Determining gestational age for public health care users in Brazil: comparison of methods and algorithm creation. BMC Res Notes 2013; 6: 60 .

11. Cascaes AM, Gauche H, Bayamarchi FM, Borges CM, Peres KG. Prematuridade e fatores associados no Estado de Santa Catarina, Brasil, no ano de 2005: análise dos dados do Sistema de Informações Sobre Nascidos Vivos. Cad Saúde Pública. 2008; 24 (5): 1024-32.

12. Matijasevich A, Silveira MF, Matos ACG, Rabello ND, Fernandes RM, Maranhão AG, Cortez-Escalante JJ, Barros FC, Victora CG. Estimativas corrigidas da prevalência de nascimentos pré-termo no Brasil, 2000 a 2011. Epidemiol Serv Saúde. 2013; 22: 557-64.

\footnotetext{
Recebido em 13 de abril de 2015

Versão final apresentada em 29 de junho de 2015

Aprovado em 15 de julho de 2015
}

13. Governo do Estado de Santa Catarina. Secretaria de Estado da Saúde. [acesso em 13 mai 2015]. Disponível em: $<$ http://www.durb.sdm.sc.gov.br/notasexplicativas.html >

14. Zhang J, Yu KF. What's the relative risk? A method of correcting the odds ratio in Cohort Studies of Common Outcomes. JAMA. 1998; 280 (19): 1690-1.

15. Barros AJ, Hirakata VN. Alternatives for logistic regression in cross-sectional studies: an empirical comparison of models that directly estimate the prevalence ratio. BMC Med Res Methodol. 2003; 3: 21.

16. Howson CP, Kinney MV, Lawn JE. Born too soon: the global action report on preterm birth. Geneva: World Health Organization; 2012.

17. França E, Lansky S. Mortalidade infantil neonatal no Brasil: Situação, tendências e perspectivas. In: Rede Interagencial de Informações para Saúde, organizador. Demografia e saúde: contribuição para análise de situação e tendências. Brasília: Organização Pan-Americana da Saúde; 2009. p. 83-112.

18. Brasil. Ministério da Saúde. Consolidação de Informações sobre os Nascidos Vivos - 2011: Coordenação Geral de Informações e Análise Epidemiológica. [acesso em $18 \mathrm{fev}$ 2015]. Disponível em:< http://tabnet.datasus.gov.br/cgi/ tabcgi.exe?SINASC/cnv/nvsc.def $>$.

19. Blencowe H, Cousens S, Chou D, Oestergaard M, Say L, Moller AB, Moller AB, Kinney M, Lawn J. Born too soon: the global epidemiology of 15 million preterm births. Reprod Health. 2013, 10 (Suppl. 1): S2.

20. Howson CP, Kinney MV, McDougall L, Lawn JE. Born too soon: preterm birth matters. Reprod Health. 2013; 10 (Suppl 1): S1.

21. Silva AA, Barbieri MA, Bettiol H, Goldani MZ, Rona RJ. Can we explain why Brazilian babies are becoming lighter? Int J Epidemiol. 2004; 33: 821-8.

22. Barros FC, Victora CG, Matijasevich A, Santos IS, Horta BL, Silveira MF, Barros AJ. Preterm births, low birth weight, and intrauterine growth restriction in three birth cohorts in Southern Brazil: 1982, 1993 and 2004. Cad Saúde Pública. 2008; 24 (Suppl. 3): S390-8. 